\title{
The prevalence of celiac disease in patients with irritable bowel syndrome
}

\author{
M. EL-SALHY ${ }^{1,2}$, B. LOMHOLT-BECK ${ }^{3}$ and D. GUNDERSEN ${ }^{4}$ \\ ${ }^{1}$ Section for Gastroenterology, Department of Medicine, Stord Helse-Fonna Hospital; ${ }^{2}$ Section \\ for Gastroenterology, Institute of Medicine, University of Bergen; ${ }^{3}$ Department of Pathology, \\ Haugesund Helse-Fonna Hospital; ${ }^{4}$ Department of Research, Helse-Fonna, Haugesund, Norway
}

Received January 26, 2011; Accepted March 7, 2011

DOI: $10.3892 / \mathrm{mmr} .2011 .466$

\begin{abstract}
The diagnosis of irritable bowel syndrome (IBS) is based on symptom assessment such as the Rome III criteria. It is sometimes difficult to clinically distinguish IBS from adult-onset celiac disease (CD). Individuals with CD presenting with relatively vague abdominal symptoms are at risk of been dismissed as having IBS. This study aimed to investigate the prevalence of patients with $\mathrm{CD}$ among those that fulfill the Rome III criteria for IBS from among patients referred to the gastroenterology section of our hospital over the last 5 years. The study included a total of 968 patients with an average age of 32 years (range 18-59 years). Females constituted 95\% of all patients. Duodenal biopsies were obtained during standard gastroscopy. Sections from these biopsies were stained with haematoxylin and eosin and immunostained for human leucocytes CD45 using the avidin-biotin complex (ABC) method. The sections were then histopathologically examined. Four patients had CD: one with Marsh type 3b, and 3 with Marsh type 1 . All four of these patients were positive for tissue transglutminase antibodies (anti-t-TG) IgA and were females aged 24, 20, 36 and 38 years. These 4 patients fulfilled the Rome III criteria for the sub-type IBS-diarrhea. This amounts to a prevalence of $0.4 \%$ of CD in IBS patients. The present findings support the notion that IBS patients should be routinely examined for CD. This applies to all subtypes of IBS.
\end{abstract}

\section{Introduction}

Approximately 15 to $25 \%$ of the world population suffers from irritable bowel syndrome (IBS) (1). IBS is a chronic condition characterized by abdominal discomfort or pain, abdominal bloating and changes in bowel habit $(1,2)$. The degree of symptoms varies in different patients from tolerable to severe,

Correspondence to: Professor Magdy El-Salhy, Section for Gastroenterology, Medicine Clinic, Stord Helse-Fonna Hospital, Box 4000, 5409 Stord, Norway

E-mail: magdy.el-salhy@helse-fonna.no

Key words: celiac disease, histopathology, irritable bowel syndrome, immunocytochemistry, prevalence, screening interfering with daily activity. IBS is the most common diagnosis in gastroenterology and is estimated to comprise $20-40 \%$ of all consultations performed by gastroenterologists $(2,3)$. Besides the increased morbidity caused by IBS, it represents an economic burden to society in different indirect forms, such as increased sick leave and over-consumption of healthcare resources $(2,3)$.

There are no biochemical, histopathological or radiological tests for the diagnosis of IBS. Instead, its diagnosis is based on symptom assessment, such as the Rome III criteria (4), and the preclusion of other diseases that cause similar symptoms. Therefore, it is sometimes difficult to clinically distinguish IBS from adult-onset celiac disease (CD) (5-10). In patients with CD presenting in adulthood, minimal or atypical symptoms are often encountered (7,9-12). The breadth of the spectrum of symptoms associated with IBS results in a potential for overlap between IBS and CD symptomatologies. Thus, individuals with $\mathrm{CD}$ presenting with relatively vague abdominal symptoms are at risk of been dismissed as having IBS (13).

The present study aimed to investigate the prevalence of CD among patients that fulfill the Rome III criteria for IBS, referred to the gastroenterology section of our hospital over the last 5 years.

\section{Materials and methods}

Patients. Patients were referred to the gastroenterology section of the Stord Helse-Fonna Hospital from December 2005 to December 2010. Those that satisfied the Rome III criteria for the diagnosis of IBS were considered for inclusion in the study. Patients who were between 18 and 60 years of age and did not suffer from organic gastrointestinal disease or clinically significant system disease were included in the study. Pregnant or lactating women were excluded. Furthermore, patients who had undergone abdominal surgery, with the exception of appendectomy, caesarean section or hysterectomy, were excluded, as were patients with a history of psychosis or mental retardation. A total of 968 patients qualified, with an average age of 32 years (range 18-59 years). Females constituted $95 \%$ of the sample. The patients underwent complete physical examination and blood tests including: full blood count, electrolytes, calcium, folic acid, vitamin $\mathrm{B}_{12}$, inflammatory markers, kidney and lever tests, and thyroid function tests. 

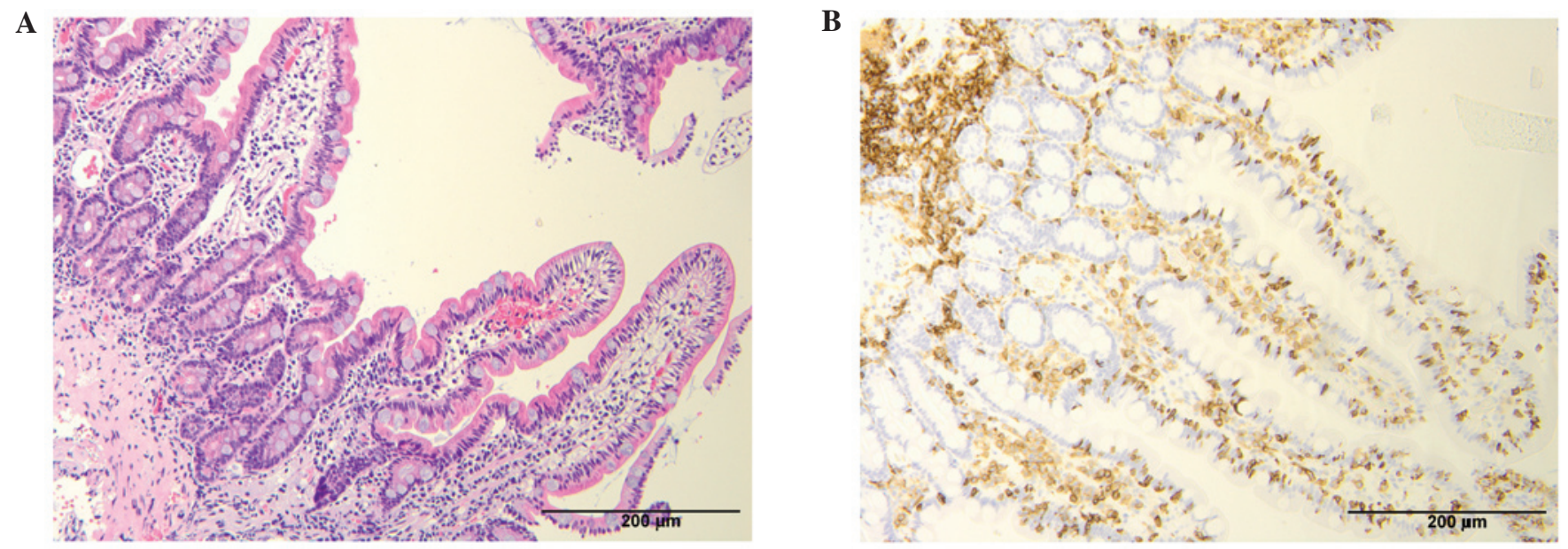

Figure 1. A duodenal biopsy from a 20-year-old female that fulfills the Rome III criteria for irritable bowel syndrome. This patient had Marsh type 1 celiac disease and was positive for tissue transglutminase antibodies (anti-t-TG) IgA. The biopsy was stained by (A) H\&E and (B) CD45.

A

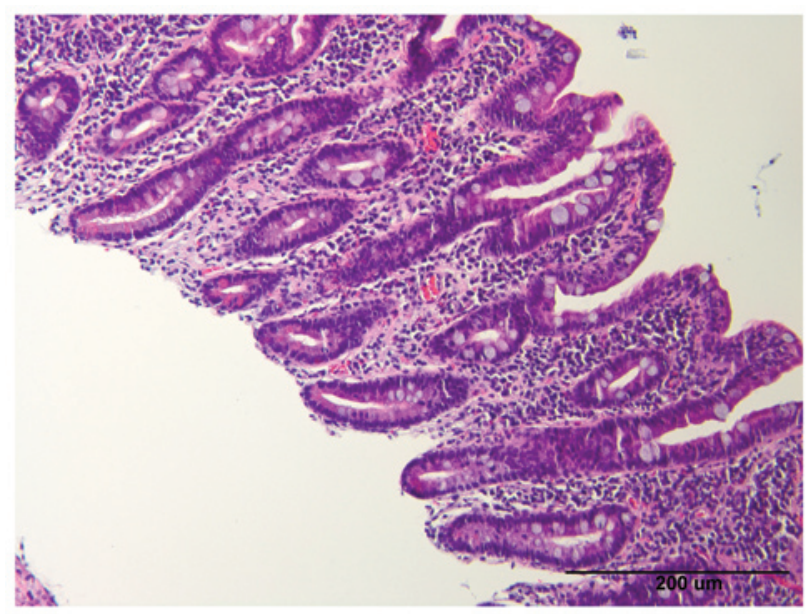

B

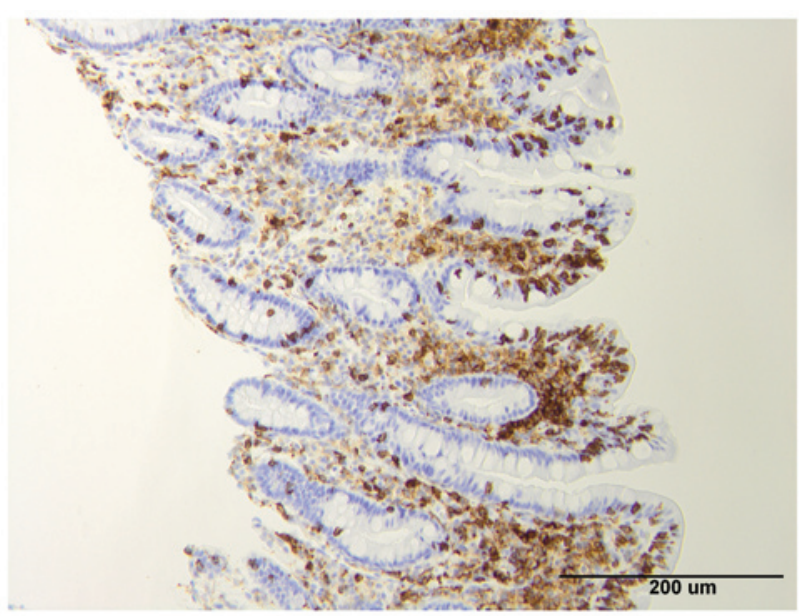

Figure 2. A duodenal biopsy from a 24-year-old female that fulfills the Rome III criteria for irritable bowel syndrome. This patient had Marsh type 3b celiac disease and was positive for tissue transglutminase antibodies (anti-t-TG) IgA. The biopsy was stained by (A) H\&E and (B) CD45.

Gastroscopy, histopathology and immunohistochemistry. After overnight fasting, a gastroscopy was performed in the patients. During the gastroscopy, four biopsies were obtained from the pars decendens duodeni (distal to the papilla of Vater). Biopsies were fixed in $4 \%$ buffered paraformaldehyde overnight, embedded in paraffin and cut into $5-\mu$ m-thick sections. The sections were stained with haematoxylin and eosin and immunostained with avidin-biotin complex (ABC) using the Vectastain ABC-kit (Vector laboratories) as described previously (14). The primary antibody used was monoclonal mouse anti-human leucocytes CD45 (Dako, no. IS751). The second layer was biotinylated mouse anti-IgG were obtained from Dako. The stained and immunostained sections were examined for the possible occurrence of CD using Marsh's modified classification.

\section{Results}

Gastroscopy, histopathology and immunohistochemistry. The oesophagus, stomach and duodenum of the patients were found to be macroscopically normal. Histopathological examination of duodenal biopsies from the patients revealed normal histology in all the patients, except for 7.CD of Marsh type 1 was found in 6 patients (Fig. 1), and CD of Marsh type $3 \mathrm{~b}$ in 1 patient (Fig. 2). The 6 patients with CD Marsh type 1 were subjected to another gastroscopy after 3-6 months and 8 new duodenal biopsies were examined. Furthermore, serological tests for tissue transglutminase antibodies (antit-TG) IgA were performed in these patients. In 3 patients with Marsh type $1 \mathrm{CD}$, the second biopsies showed normal histology and negative anti-tTG IgA. These patients were a male and 2 females aged 36, 22 and 42 years, respectively. The remaining 3 patients with Marsh type 1 showed the same histopathological changes in the second duodenal biopsies and were positive for anti-t-TG IgA. Thus, 4 patients had a certain diagnosis of CD among the 968 investigated. All 4 were females aged 24, 20,36 and 38 years. These 4 patients fulfilled the Rome III criteria for the sub-type IBS-diarrhoea. This amounts to a prevalence of $0.4 \%$ of celiac disease in IBS patients. 


\section{Discussion}

IBS is prevalent in $15-25 \%$ of the world population, while the prevalence of CD is only $0.5-1 \%$ (15-18). There is, however, an overlap in the symptoms between IBS and CD. Since the diagnosis of IBS is based mainly on symptom assessment, there is a risk of CD patients being wrongly diagnosed as having IBS. This risk is even higher in the two IBS subtypes: IBS-diarrhoea and IBS-mixed. The situation is further complicated by the fact that the abdominal symptoms of both CD and IBS patients are triggered by the ingestion of wheat products. In CD patients, this is due to gluten allergy, while in IBS, the effect is attributed to the long sugar polymer fructan in the wheat (19).

Three previous studies have reported a $0 \%$ prevalence of $\mathrm{CD}$ in IBS patients, while in another two studies the prevalence was found to be 11.4 and $31.8 \%$, respectively (20-24). These studies were performed on a small patient sample, and therefore definite conclusions could not be drawn. The reported prevalence of $\mathrm{CD}$ in IBS patients varies among different studies, in the range of $0.7-4.7 \%(18,25-28)$. In the present study, the prevalence of CD among IBS patients was found to be $0.04 \%$. Notably, we used small intestinal biopsies and histopathological examination for CD detection, which is the golden standard. In most of the previous studies, serological tests were instead used for screening.

Regardless of the number of the CD patients among patients diagnosed with IBS, IBS patients should be routinely examined for CD, applicable to all subtypes of IBS. This is evidenced by the finding that $2.6 \%$ of patients diagnosed with the IBS-constipation subtype have been found to have CD (18). As to which screening approach should be used, both serological and small intestinal biopsies have their advantages and disadvantages; thus, the method that works best locally should be implemented.

\section{Acknowledgements}

The authors wish to thank Ann Kristin Koppang for her enthusiasm and for assisting with and maintaining the patient lists. Thanks are also due to Åsa Helene Lundal at the Department of Pathology, Haugesund Hospital, for co-ordination of the collaboration between the Stord and Haugesund Hospitals. We would like to express our gratitude to Professor Hans Olav Fadnes, head of the Department of Medicine, Stord HelseFonna Hospital, for his support and for reading the manuscript. This study was supported by a grant from Helse-Fonna.

\section{References}

1. Thompson WG: A world view of IBS. In: Irritable Bowel Syndrome: Diagnosis and Treatment. 1st edition. Camilleri $\mathrm{M}$ and Spiller R (eds). Saunders, New York, NY, pp17-26, 2002.

2. Everhart JE and Renault PF: Irritable bowel syndrome in officebased practice in the United States. Gastroenterology 100: 998-1005, 1991.

3. Harvey RF, Salih SY and Read AE: Organic and functional disorders in 2000 gastroenterology outpatients. Lancet 1: 632-634, 1983.

4. Longstreth GF, Thompson WG, Chey WD, Houghton LA, Mearin F and Spiller RC: Functional bowel disord. Gastroenterology 130: 1480-1491, 2006.

5. Fissora $\mathrm{Cl}$ and Koch KL: Symptom overlap and comorbidity of irritable bowel syndrome with other conditions. Curr Gastroenterol Rep 7: 264-271, 2005.
6. Sanders DS, Carter MJ, Hurlstone DP, Pearce A, Ward AM, McAlindon ME and Lobo AJ: Association of adult coeliac disease with irritable bowel syndrome: a case-control study in patients fulfilling ROME II criteria referred to secondary care. Lancet 358: 1504-1508, 2001

7. Ziper RD, Patel S, Yahya KZ, Baisch DW and Monarch E: Presentations of adult celiac disease in nationwide patient support group. Dig Dis Sci 48: 761-764, 2003.

8. Wahnscaffe U, Ulrich R, Rieken EO and Sculzke JD: Celiac disease-like abnormalities in subgroup of patients with irritable bowel syndrome. Gastroenterology 121: 1329-1338, 2001.

9. Bottaro G, Calado F, Rotolo N, Spina M and Corazza GR: The clinical pattern of subclinical/silent celiac disease: an analysis on 1026 consecutive cases. Am J Gastroenterolo 94: 691-696, 1999.

10. Green PHR, Stavropoulos SN, Panagi SG, Goldstein SL, McMahon DJ, Absan H and Neugut AI: Characteristics of adult celiac disease in the USA: results of a national survey. Am J Gastroenterol 96: 126-131, 2001.

11. Makharia GK, Baba CS, Khadgawat R, Lal S, Tvaatia MS, Madan K and Dattagupta S: Celiac disease: variations of presentation in adults. Indian J Gastroenterol 26: 162-106, 2007.

12. Lo W, Sano K, Lebwohl B, Diamond B and Green PH: Changing presentation of adult celiac disease. Dig Dis Sci 48: 395-398, 2003.

13. Morcos A, Dinan T and Quigley NM: Irritable bowel syndrome: role of food in pathogenesis and management. J Dig Dis 10: 237-246, 2009.

14. El-Salhy M, Stenling R and Grimelius L: Peptidergic innervation and endocrine cells in the human liver. Scand J Gastroenterol 28: 809-815, 1993.

15. Catassi C, Fabian E, Rätsch IM, et al: The celiac iceberg in Italy. A multicentre antigliadin antibodies screening for celiac disease in school-age subjects. Acta Paediatr 412 (Suppl): 29-35, 1996.

16. Dubè C, Rostom A, Sy R, et al: The prevalence of celiac disease in average risk Western European populations: a systematic review. Gastroenterology 128: S57-S67, 2005.

17. Not T, Horvath K, Hill ID, Partanen J, Hammed A, Magazzu G and Fasano A: Celiac disease risk in the USA: high prevalence of antiendomysium antibodies in healthy blood donors. Scand J Gastroenterol 33: 494-498, 1998.

18. Fasano A, Berti I, Geraduzzi, et al: Prevalence of celiac disease in at-risk and not-at-risk groups in the USA: a large multicentre study. Arch Intern Med 163: 286-292, 2003

19. Heizer WD, Southern S and McGovern S: the role of diet in symptoms of irritable bowel syndrome in adults: a narrative review. J Am Diet Assoc 109: 1204-1214, 2009.

20. Wouden E, Nelis JF and Vecht J: Screening for coeliac disease in patients fulfilling the Rome II criteria for irritable bowel syndrome in a secondary care hospital in The Netherlands: a prospective observational study. Gut 56: 444-445, 2007.

21. Locke GR, Murry JA, Zinsmeister AR, et al: Celiac disease serology in irritable bowel syndrome and dyspepsia: a populationbased case-control study. Mayo Clin Proc 79: 476-482, 2004.

22. Hin H, Bird G, Fisher P, et al: Celiac disease in primary care: case finding study. BMJ 318: 164-167, 1999.

23. Shahbazkhani B, Forootan M, Merat S, et al: Celiac disease presenting with symptoms of irritable bowel syndrome. Aliment Pharmocol Ther 18: 231-235, 2003.

24. Catassi C, Kryszak D, Louis-Jacques O, et al: Detection of celiac disease in primary care: a multicenter case-finding study in North America. Am J Gastroenterol 102: 1454-1460, 2007.

25. Sanders DS, Carter MJ, Huristone DP, Pearce A, Ward AM, McAlindon ME and Lobo AJ: Association of adult coeliac disease with irritable bowel syndrome: a case-control study in patients fulfilling ROME II criteria referred to secondary care. Lancet 358: 1504-1508.

26. Korkut E, Bektas M, Oztas E, Kurt M, Cetinkaya H and Ozden A: The prevalence of celiac disease in patients fulfilling Rome III criteria for irritable bowel syndrome. Eur J Intern Med 21: 389-392, 2010.

27. Sanders DS, Patel D, Stephenson TJ, et al: A primary care cross-sectional study of undiagnosed adult coeliac disease. Eur J Gastroenterol Hepatol 15: 407-413, 2003.

28. Holt R, Darnley S, Kennedy $\mathrm{T}$ and Jones R: Screening for coeliac disease in patients with clinical diagnosis of irritable bowel syndrome. Gastroenterology 120: A757-A808, 2001. 